\title{
Liquid Intrusion into Zeolitic Imidazolate Framework-7 Nanocrys- tals: Exposing the Roles of Phase Transition and Gate Opening to Enable Energy Absorption Applications
}

\author{
Yueting Sun, ${ }^{\dagger}$ Yibing Li, ${ }^{\ddagger}$ and Jin-Chong Tan ${ }^{*} \dagger$ \\ ${ }^{\dagger}$ Multifunctional Materials \& Composites (MMC) Laboratory, Department of Engineering Science, University of Oxford, \\ Parks Road, Oxford OX1 3PJ, United Kingdom \\ "State Key Laboratory of Automotive Safety and Energy, Tsinghua University, Beijing, 100084, P.R. China
}

\begin{abstract}
Liquid intrusion into zeolitic imidazolate framework 7 (ZIF-7) has been observed for the first time. Amongst the three typical phases of ZIF-7, we discover that only the guest-free ZIF-7-II structure can be intruded by mechanical pressure, and intriguingly, this pressurized liquid intrusion behavior is detected only in nanocrystals indicating the crystal size effect. Because of its unique combination of non-outflow property and high intrusion pressure, water intrusion into ZIF-7-II generates a marked energy dissipation capacity of $\sim 2 \mathrm{~J} / \mathrm{g}$ despite its limited pore volume. We present several strategies that can be easily implemented to tune its intrusion pressure and energy dissipation, and to accomplish material reusability. Remarkably, we found that the pore cavities of ZIF-7-II can accommodate water molecules without experiencing any phase transition, which is entirely different from other solvents whose incorporation will trigger a spontaneous conversion into ZIF-7-I. Our pressure-vs-volume data further reveal that the process of water infiltration and retainment is controlled by the gate-opening/-closing mechanism, which has enabled us to probe the viscoelasticity of ZIF-7 via cyclic liquid intrusion experiments. This study has deepened our understanding of the time-dependent mechanical properties of ZIFs and shed new light on structural flexibility central to the novel applications of metal-organic framework materials.
\end{abstract}

Keywords: zeolitic imidazolate framework, liquid intrusion, hydrophobicity, gate opening, intrusion pressure, phase transition.

\section{INTRODUCTION}

Tunable nanofluidic platforms are of considerable current interest due to their wide ranging technological applications, encompassing targeted drug delivery and cell therapy, lab-onchips, and fine-scale energy conversion devices. ${ }^{1-3}$ Particularly, understanding the pressure-induced liquid intrusion behavior into nanoporous materials is receiving increasing attention, ${ }^{4-5}$ motivated by its unconventional energy conversion mechanisms. ${ }^{6-7}$ Nanoscale solid-liquid interaction of ultra-large surface area promises a high conversion efficiency between the mechanical, interfacial, and thermal energies. ${ }^{8-9}$ Nevertheless, the current efforts are by and large limited to nanofluidics of non-flexible (i.e. structurally rigid) nanostructures and of larger sized nanopores (compared to the molecular size of liquid)..$^{10-11}$ It is fundamentally important to understand the basic behavior underpinning the pressurized liquid flow within molecularsized and deformable structures, ${ }^{12}$ because the single file transport of liquid molecules is far from obeying the classical continuum theories; for instance, it may exhibit superfast transport rates. ${ }^{13-14}$ Moreover, the nanoscale confinement effects conferred by the geometry of nanosized channels could strongly influence the nanofluidic response of the system as a whole. ${ }^{15-16}$

In the light of this, liquid intrusion into zeolitic imidazolate framework (ZIF), specifically ZIF-7, has been explored in this paper. ZIFs are crystalline nanoporous materials constructed from tetrahedral metal centers (e.g. $\mathrm{Zn}^{2+}$ or $\mathrm{Co}^{2+}$ ) bridged by imidazolate-type organic linkers. They have flexible openframework structures due to the coordinative metal-imidazolate bonding, in contrast to their aluminosilicate analogues, zeolite, whose structures are mechanically more rigid. ${ }^{17-18}$ For this reason, it is projected that liquid intrusion may give rise to structural transformation of ZIFs.

ZIF-7 [Zn(bIM $)_{2}$, bIM = benzimidazolate] is amongst the earliest reported structures within the ZIF family of metal-organic frameworks (MOFs). It has a sodalite (SOD) framework topology, which is identical to that of the ZIF-8. However, ZIF-7 has a very narrow channel aperture (i.e. pore window opening connecting adjacent sodalite cages) reported as $2.9 \AA$ (vs. $3.4 \AA$ in ZIF-8) ${ }^{19}$ whose size is comparable with the kinetic diameter of water $(2.7 \AA) .{ }^{20}$ Given its constricted channels, ZIF-7 is widely regarded as a promising candidate for hydrogen-selective membranes. ${ }^{21}$ Because of the gate-opening mechanism of ZIF-7, it is effective for separating hydrocarbon molecules of very similar sizes. ${ }^{22}$ To the best of our knowledge, this will be the narrowest aperture size yet for which pressurized liquid intrusion has been targeted. We note that although water intrusion into ZIF-7 has been attempted in a recent study, its liquid intrusion behavior has not yet been successfully detected. ${ }^{23}$ Our results present the first unambiguous observation of liquid intrusion into the ZIF-7 nanocrystals, carefully taking into consideration its three distinct phases (scheme 1) and the salient effects of crystal size on the liquid intrusion response. 
Scheme 1. Phase transitions of ZIF-7 phase -I, -II and -III

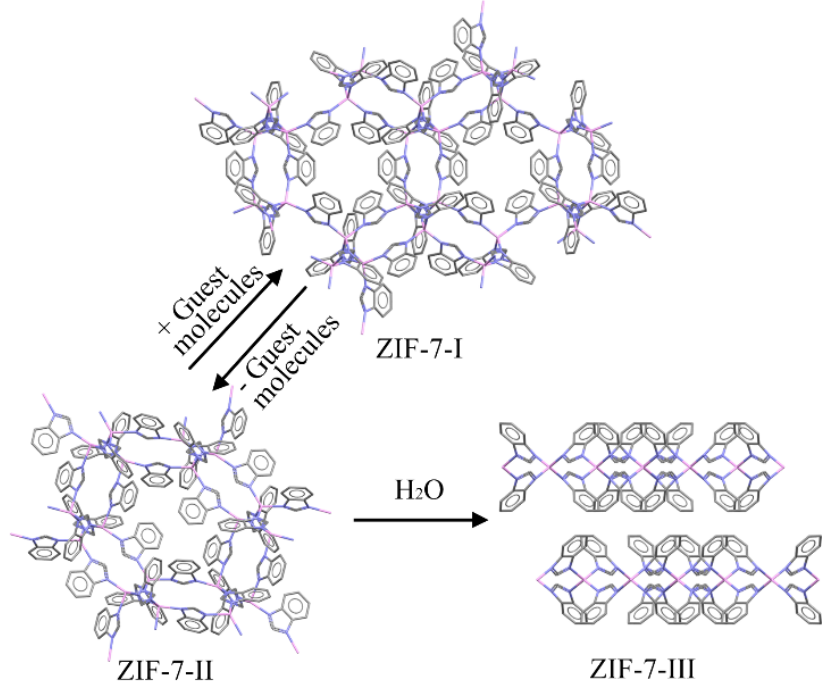

As shown in scheme 1, it is important to recognize that ZIF-7 can adopt multiple phases, where precise knowledge of its phase transition is important as this may affect its sorption and mechanical properties. ${ }^{24-25}$ The as-synthesized ZIF-7 material typically has occluded guest solvent molecules (e.g. $N, N$-dimethylformamide (DMF)) in its pores, this phase has a rhombohedral unit cell ( $R \overline{3}$ spacegroup) and is designated as the ZIF-7-I. The removal of guest molecules from the pores of ZIF-7-I will result in the formation of the second phase, termed ZIF-7-II with a triclinic unit cell ( $P \overline{1}$ spacegroup). Such a phase transition from ZIF-7-I to ZIF-7-II can be achieved via heat treatment, or reversed through immersion in solvents to yield guest incorporation. The third phase, called ZIF-7-III has a monoclinic unit cell ( $C 2 / c$ spacegroup). ZIF-7-III features a two-dimensional (2D) layered architecture, ${ }^{26}$ comprising four-membered rings of corner-shared networks of $\mathrm{Zn}$ (II) benzimidazolate tetrahedra.

In this work, we examine the liquid intrusion behavior of all the three phases of ZIF-7 (scheme 1) subject to a mechanical pressure. Particularly, we interrogate the structural deformation and gate opening of ZIF-7 associated with pressure-induced infiltration by water molecules. We characterize the tunability of the ZIF-7+water system, to determine its mechanical performance and potential for engineering practical applications.

\section{MATERIALS AND METHODS}

ZIF-7 nanocrystals were synthesized in accordance with the procedures reported by Li et al. ${ }^{27}$ The as-synthesized product is the DMF-incorporated ZIF-7-I phase, confirmed via powder Xray diffraction (PXRD in Figure 1a). The average particle size is $\sim 30 \mathrm{~nm}$ as estimated from scanning electron microscopy (SEM; see Figure S1 in the supporting information (SI)). The ZIF-7-II phase was obtained by removal of the DMF molecules from ZIF-7-I at $200{ }^{\circ} \mathrm{C}$ in air for $12 \mathrm{~h}$. According to the XRD data, the consequent ZIF-7-II exhibits a structural distortion with symmetry loss (from $R \overline{3}$ to $P \overline{1}$, see scheme 1 ). Compared with the reported experimental XRD pattern of ZIF-7-II (from ref.24 which is based on micron-sized ZIF-7-II crystals), here it is evident that the broadening of the Bragg peaks is due to the nano-sized crystals, also evidenced in a previous study, where an identical XRD pattern was obtained for ZIF-7-II with a crystal size $\sim 100 \mathrm{~nm}^{21}$ The reversed phase transition from the guestfree ZIF-7-II to solvent-occluded ZIF-7-I will be discussed in the following sections. Finally, we found that the 2-D layered ZIF-7-III phase can be obtained by simply immersing ZIF-7-II in water for one week. Because the above phase transition is a time-dependent process, an intermediate phase can be expected if the immersion period is to be shortened.

In order to further validate that the correct phases of ZIF-7 are obtained, Fourier-transform infrared (FT-IR) and Raman spectroscopies were conducted. The IR spectra are shown in Figure S2 (SI), the three phases share the same major absorption bands, e.g., the band at $740 \mathrm{~cm}^{-1}$ associated with the out-ofplane $\mathrm{C}-\mathrm{H}$ bending of the benzene ring, and the peaks around $3050 \mathrm{~cm}^{-1}$ corresponding to the $\mathrm{C}-\mathrm{H}$ stretching of imidazolate ring. ${ }^{28-29}$ By comparing amongst the different phases, the IR spectra evidenced the successful removal of DMF molecules from ZIF-7-I to yield ZIF-7-II. The peaks corresponding to DMF molecules inside ZIF-7-I are absent in ZIF-7-II, indicating that we achieved the correct guest-free phase: ZIF-7-II. ZIF7-III can also be differentiated from the other two phases by the IR spectra. Many peaks are shifted to either a lower or higher wavenumber (e.g., those in the region of $1100-1200 \mathrm{~cm}^{-1}$ ), which should be associated with the change in the strength (and interatomic spacing) of chemical bonds. It can be seen that some new peaks also split out upon the transition from ZIF-7-II to ZIF-7-III, for example at $753 \mathrm{~cm}^{-1}$ and $1478 \mathrm{~cm}^{-1}$.

The Raman spectra are shown in Figure S3 (SI), with the bands assigned according to ref.24. Notable are the Raman bands corresponding to the vibrational modes of the benzimidazolate linker $\left(\sim 550 \mathrm{~cm}^{-1}\right)$ and the terahertz modes of the framework lattice $\left(\sim 50-150 \mathrm{~cm}^{-1}, c a .1-5 \mathrm{THz}\right)$. A structural distortion from ZIF-7-I to ZIF-7-II is responsible for the significant distinction in the regions corresponding to the lattice modes, the torsion modes of the imidazolate ring, and the in-plane bending modes of the bridging linkers. In the same regions, ZIF-7-III also exhibits distinctive Raman bands from the other two phases, attributable to its different framework structure (2D).

For further verification, three phases of micron-sized ZIF-7 crystals were also synthesized using similar methods (but a higher ratio of metal to linker and higher reaction temperature, see methods in SI). ${ }^{30}$ Due to the bigger crystal size $(\sim 3 \mu \mathrm{m}$, see SEM in Figure S4a), the micron-sized ZIF-7-II has a better quality XRD pattern compared to the nanocrystals (Figure $\mathrm{S} 4 \mathrm{~b}$ vs. Figure 1a). Their IR and Raman spectra are also included in Figure S2 and S3, but no significant difference can be distinguished between the nano- and micron-sized crystals, for any of the three phases. Thus ZIF-7 nanocrystals have exactly the same chemical structure and functional group as the micron-sized crystals. Despite the broadening of Bragg peaks in Figure 1a due to the nano-sized crystals, we confirmed that the correct guest-free ZIF-7-II phase has been employed for this study.

For the liquid intrusion experiments, $25 \mathrm{mg}$ of the ZIF-7 powder was mixed with $0.1 \mathrm{~mL}$ water, then sealed in a stainless steel chamber with precisely-fitted sealing rings (see Figure S5 in SI). Using the Instron 5582 universal testing machine, a piston was compressed into the chamber at a constant loading rate of $0.5 \mathrm{~mm} / \mathrm{min}$. Once the pressure of the system has reached the 
maximum value of $106 \mathrm{MPa}$, the movement of the piston was reversed at $-0.5 \mathrm{~mm} / \mathrm{min}$ during the unloading stage.

\section{WATER INTRUSION BEHAVIOR OF ZIF-7}

Figure $1 \mathrm{~b}$ shows the recorded $P-\Delta V$ curves (i.e. applied pressure versus volumetric change plot) to characterize water intrusion into the three phases of ZIF-7 nanocrystals. It can be seen that, both ZIF-7-I and ZIF-7-III exhibit a purely elastic linear behavior with negligible hysteresis, indicating that there is no accessible framework porosity to afford water intrusion. For ZIF-7-I, this is simply because the DMF guest molecules are still blocking the pore volume within the sodalite cages. Interestingly, for ZIF-7-III, our data show that the narrow gap between the 2D interlayers (bound by van der Waals interactions) cannot be intruded by water molecules; therefore ZIF-7-III has no accessible porosity and no plateau was observed in its $P-\Delta V$ curve.
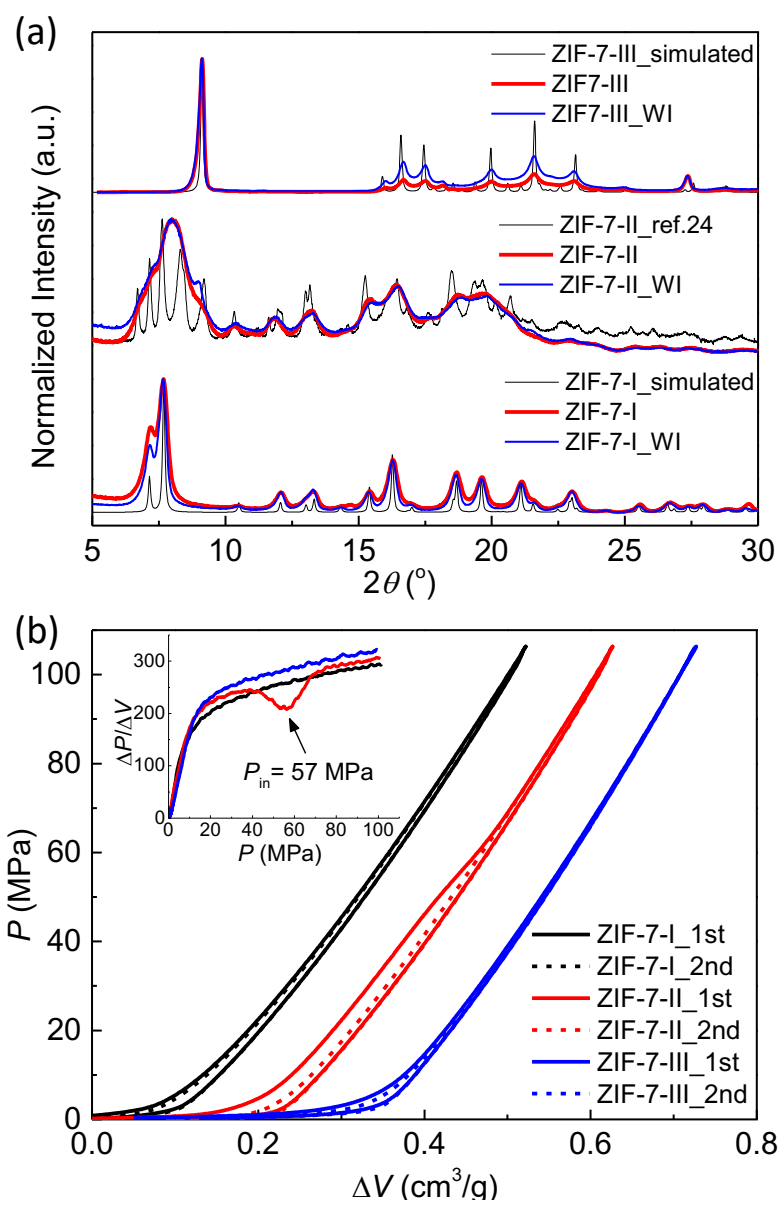

Figure 1. (a) XRD patterns of the three phases of ZIF-7 nanocrystals, before and after water intrusion (marked with 'WI') experiments. The simulated XRD is obtained from ref.31 (ZIF-7-I) and ref.26 (ZIF-7-III) respectively. The simulated structure of ZIF-7-II is available in ref.24 but it is of poor quality (see Figure S6 in SI), therefore it is more meaningful to apply the reported experimental data in ref.24 for direct comparison to our data. (b) $P-\Delta V$ curves of water intrusion into the three phases of ZIF-7 nanocrystals, including two consecutive loading cycles (denoted as 1 st and $2 \mathrm{nd}$, respectively). The inset shows the gradient $\Delta P / \Delta V$ of the loading part of the first cycle, from which the intrusion pressure $P_{\text {in }}=57 \mathrm{MPa}$ was found. Note: $P-\Delta V$ curves have been shifted horizontally for clarity.
Significantly, for the first time, we have succeeded in detecting the water intrusion of ZIF-7-II. According to its $P-\Delta V$ curve in Figure $1 b$, the pressure first increases linearly with volume change, because ZIF-7 is hydrophobic and rejects water molecules located outside the nanopores. When the external pressure has been raised to $57 \mathrm{MPa}$, the capillary effect is surpassed and water molecules begin to intrude the ZIF-7-II framework. Consequently, a plateau is observed here associated with this water intrusion phenomenon, which deviates from the elastic modulus of the system. Eventually when all of the pores have been filled up by water molecules, the plateau ends and turns to a linear behavior again. For improved analysis of the loading process, the inset of Figure $1 \mathrm{~b}$ presents the gradient $\Delta P / \Delta V$ as function of $P$, where the intrusion pressure $P_{\text {in }}$ (corresponds to the height of intrusion plateau) can be established when $\Delta P / \Delta V$ shows a turning point.

Moreover, we discovered that by applying different heating conditions on ZIF-7-I, the resulting ZIF-7-II phase will exhibit varying water intrusion pressures. As shown in Figure S7 (SI), the intrusion pressure increases with a higher heat treatment temperature or longer duration; while no significant difference can be identified through the XRD patterns of the three ZIF-7-II samples. In order to gain more insights, we obtained the FT-IR and Raman spectra of these three ZIF-7-II samples (see Figure S8-S9 in SI), which are almost identical in general. However, a closer observation reveals that there might be a little amount of DMF molecules left inside the ZIF-7 framework if the most moderate heating treatment is adopted $\left(153^{\circ} \mathrm{C}\right.$ for $\left.3 \mathrm{~h}\right)$, indicated by the tiny hump in FT-IR spectra around $1675 \mathrm{~cm}^{-1}$ (Figure $\mathrm{S} 8 \mathrm{~b})$ which is not present for the other two cases. In addition, the ZIF-7-II sample obtained by heating at $330^{\circ} \mathrm{C}$ for $36 \mathrm{~h}$ seems to have some minor differences in Raman spectra from the other two samples (e.g., the lattice vibrations at $50-150 \mathrm{~cm}^{-1}$, see Figure S9b), indicating the possibility of structural change. This might be the reason for the different intrusion pressures caused by varying heating conditions. However, it could also be attributed to the modification of the mechanical properties and/or hydrophobicity of ZIF-7-II resulting from the different heat treatments; the detailed mechanism is not yet understood and needs to be explored further. Moreover, despite the thermal stability of ZIF-7, it is worth noting that very intensive heat treatment on ZIF-7 (e.g., $480{ }^{\circ} \mathrm{C}$ for $12 \mathrm{~h}$ ) will result in its structural decomposition into zinc oxide, as shown in Figure S10 (SI).

Some MOF materials have breathing effect due to framework flexibility, that is, their structures will shrink under mechanical pressure, whereby one can also observe plateaus in their $P-\Delta V$ curves. ${ }^{32-33}$ In light of this, we performed a hydrostatic compression test on ZIF-7-II nanocrystals using silicone oil as the pressure transmitting liquid. Silicone oil cannot penetrate the small nanopores of ZIF-7, and therefore is a good candidate to examine the possibility of structural breathing of ZIF-7 under mechanical pressure. As shown in Figure S11 (SI), no plateau can be observed upon the compression by silicone oil, even when the hydrostatic pressure exceeds $200 \mathrm{MPa}$. This result validates that the observed plateau in the water intrusion test (Figure 1b) is indeed caused by the penetration of water molecules into the ZIF-7-II framework, rather than the compressive deformation of the framework from breathing effect. 
The unloading curve of ZIF-7-II in Figure $1 \mathrm{~b}$ is a linear recovery, indicating the retention of water molecules (i.e. no extrusion) upon the release of the externally applied pressure. Because of that, the subsequent loading cycle (dash lines in Figure 1b) exhibits no plateau due to the loss of porosity after liquid intrusion. This is an important discovery: the non-outflow phenomenon evidenced in ZIF-7 distinguishes it from the rest of the ZIF compounds explored to date, ${ }^{23,33}$ all of which show an outflow of water molecules once the external pressure has been reduced to a certain level. For instance, ZIF-8 (Basolite Z1200, BASF) can be intruded by water at $25 \mathrm{MPa}$ and extruded at 18 $\mathrm{MPa},{ }^{34}$ see Figure S12 (SI) contrasting the water intrusion performance of ZIF-7-II against that of ZIF-8. The intrusion-extrusion behavior of ZIF-8 indicates that it is deforming in a 'superelastic' manner, thus the energy being dissipated within one loading-unloading cycle is rather limited despite it possessing a considerably larger specific pore volume (vide infra).

Surprisingly, although the accessible pore volume of ZIF-7-II is markedly lower than that of ZIF-8 $\left(30 \mathrm{~mm}^{3} / \mathrm{g}\right.$ compared with $400 \mathrm{~mm}^{3} / \mathrm{g}$, determined from the length of the intrusion plateau of the $P-\Delta V$ curves), because of its remarkable non-outflow characteristics combined with a relatively higher intrusion pressure (57 MPa compared with $25 \mathrm{MPa}$ for ZIF-8), ZIF-7-II has an energy dissipation density of $2.11 \mathrm{~J} / \mathrm{g}$ via pressurized water intrusion, which is notable against the value of ZIF-8 $(2.85 \mathrm{~J} / \mathrm{g})$. In the following sections, we will show that the energy density of ZIF-7-II can be enhanced further, and its recoverability can also be achieved by tuning the liquid properties. It is noted that the intrusion pressure of ZIF-7-II obtained here is reasonable compared with reported results of certain zeolites ${ }^{35-36}$ and other ZIFs including ZIF-8, -67, -71 (reported at tens of MPa). ${ }^{23,33}$

We found that the ZIF-7-II materials can be regenerated by evacuating the intruded water from the framework for reuse. Figure S13 (SI) presents the re-intrusion results of recycled ZIF-7-II, recovered by heating the sample at $120^{\circ} \mathrm{C}$ overnight under vacuum. The recycled ZIF-7-II regains the porosity for subsequent water intrusion, evidenced by the dip in the gradient $\Delta P / \Delta V$ (Figure $\mathrm{S} 13 \mathrm{a}$ ). There is a slight decline in its intrusion volume compared with the fresh material, indicating that the evacuation condition might be further improved. No phase transition is detected, however, throughout the water intrusion and recycling process (Figure $\mathrm{S} 13 \mathrm{~b}$ ).

We reasoned that the unique non-outflow phenomenon witnessed in ZIF-7-II can be ascribed to the very narrow channel apertures $(\sim 1.6 \AA$, see Figure S14 in SI) and flexibility of its cage structure. Since the kinetic diameter of the water molecule $(2.7 \AA)^{20}$ is much larger than the channel aperture size, the ZIF-7-II framework has to open the 'gates' (via rotation of the bIM linkers $)^{30,37}$ to allow water molecules into the sodalite cage during loading. Upon unloading, the confined water molecules have to reopen the narrow apertures in order to escape from the cage. This gate-opening mechanism can be driven by hydraulic pressure during the loading stage. However, during unloading the confined water molecules may not have sufficient free energy to re-open the gates. Consequently, water molecules become trapped inside the ZIF-7-II pores, akin to a stable 'energy well' for the system. In contrast, other ZIFs (e.g., ZIF-8, ZIF-67, ZIF-71 whose water intrusion behavior have been studied $)^{23}$ have a larger channel aperture $(3.4 \AA, 3.4 \AA, 4.2 \AA$ respec- tively) ${ }^{19}$ and all of which greater than the size of a water molecule, this may explain why they exhibit a complete water outflow upon unloading.

We have conducted re-intrusion tests on ZIF-7-II nanocrystals after an interval of $24 \mathrm{~h}$ and $84 \mathrm{~h}$ respectively from the first loading cycle (Figure S15 in SI). No intrusion plateau can be detected, indicating that the intruded water molecules are still trapped inside the ZIF-7-II framework, without any outward diffusion during the prolonged relaxation time although the external pressure has been removed. A rough estimate of the degree of gate-opening can be obtained by simply comparing the initial aperture size of ZIF-7-II with the water molecule size. However, it may also dependent on the interaction between liquid molecules and the nanopores, the spatial orientation of the liquid molecules while entering the pores, and the thermal vibration of the liquid molecules, etc. Molecular dynamics simulations can be a way forward to visualize the gate-opening of ZIF-7 in the interaction with liquid molecules. Experimentally, a viable method is to use different liquid molecules to probe the aperture of ZIF-7, to test if they can penetrate. Based on the results of a number of different liquids, the extent of gate-opening can be estimated; this can be a future work in this area.

The XRD data in Figure 1a show that ZIF-7-II retains the same phase after water intrusion, indicating that the incorporation of water molecules does not induce significant structural deformation (strains) to the structure. This is likely due to the sufficient pore volume to accommodate water molecules, and the absence of strong chemical interactions between water and the hydrophobic framework. A recent study by Cai et al. ${ }^{21}$ has tried to explore the phase transition of ZIF-7-II upon the incorporation of water molecules (through water vapor adsorption or liquid immersion), but no phase transition was observed due to the lack of driving force to enable penetration of water molecules. While in this work, by employing the pressurized liquid intrusion method, we report the first evidence that water inclusion will not transform ZIF-7-II to ZIF-7-I as other solvents do. Furthermore, the XRD results in Figure 1a also show that ZIF-7-I retains its phase after water intrusion, suggesting that there is no guest-exchange between water and DMF molecules during the liquid intrusion test, otherwise the phase transition from ZIF-7-I to ZIF-7-II would have been detected.

For a further analysis, we conducted atomic force microscopy (AFM) imaging on the ZIF-7 nanocrystals after water intrusion, and compared with the fresh samples. As shown in Figure S16 (SI), no significant changes in the crystal morphology can be identified as a result of water intrusion, for any of the three phases. Despite the inevitable aggregation of nanocrystals due to the drying or heating process during sample preparation, the nanocrystal features are still evident for all the samples examined. FT-IR and Raman spectroscopies for the water-intruded ZIF-7 nanocrystals are also obtained (see Figure S17 and S18 in SI). No difference can be detected in comparison with the spectra of the pristine samples. All the above experiments show that ZIF-7 material, in any of its three phases, is stable against water intrusion. ZIF-7 has maintained its initial molecular structure, chemical groups, as well as crystal morphology after water intrusion.

\section{INTRUSION BY ALCOHOLS \& SIZE EFFECTS}


In light of the fact that the accommodation of DMF (with a kinetic diameter of $5.5 \AA)^{38}$ can transform ZIF-7-II to ZIF-7-I, while the intrusion and retention of water $(2.7 \AA)$ cannot, we systematically investigated this effect by employing a series of alcohols with a molecule size ranging from $2.7 \AA$ to $5.5 \AA$, namely methanol $(\mathrm{C} 1,3.6 \AA)$, ethanol ( $\mathrm{C} 2,4.5 \AA)$, isopropanol $(\mathrm{C} 3,4.7 \AA)$, butanol $(\mathrm{C} 4,5.0 \AA)$ and pentanol $(\mathrm{C} 5,5.0 \AA) .{ }^{20}$ Interestingly, our results show that the incorporation of all of these alcohols (by immersion) can transform ZIF-7-II into ZIF-7-I (Figure 2a), and the ZIF-7-I obtained does not allow water intrusion or guest-exchange with water molecules (Figure $2 b)$. Therefore in a geometric sense, the upper limit of the molecular size that ZIF-7-II can accommodate without experiencing structural transformation is between $2.7 \AA$ and $3.6 \AA$. But inevitably, the chemical interaction between guest molecules and ZIF-7 structure will also play a role. The only distinction between the above solvents is their differential uptake and diffusion response. Unlike large molecules such as DMF that takes a long immersion time to enter the pores, smaller guests like methanol can trigger the phase transition immediately. And we also found that the resultant ZIF-7-I (DMF) is much more stable than ZIF-7-I (MeOH). Methanol can diffuse out easily, ${ }^{39}$ and therefore the two consecutive XRD scans of ZIF-7 (MeOH) in Figure 2a reveal the evolution from ZIF-7-I to ZIF-7-II patterns.
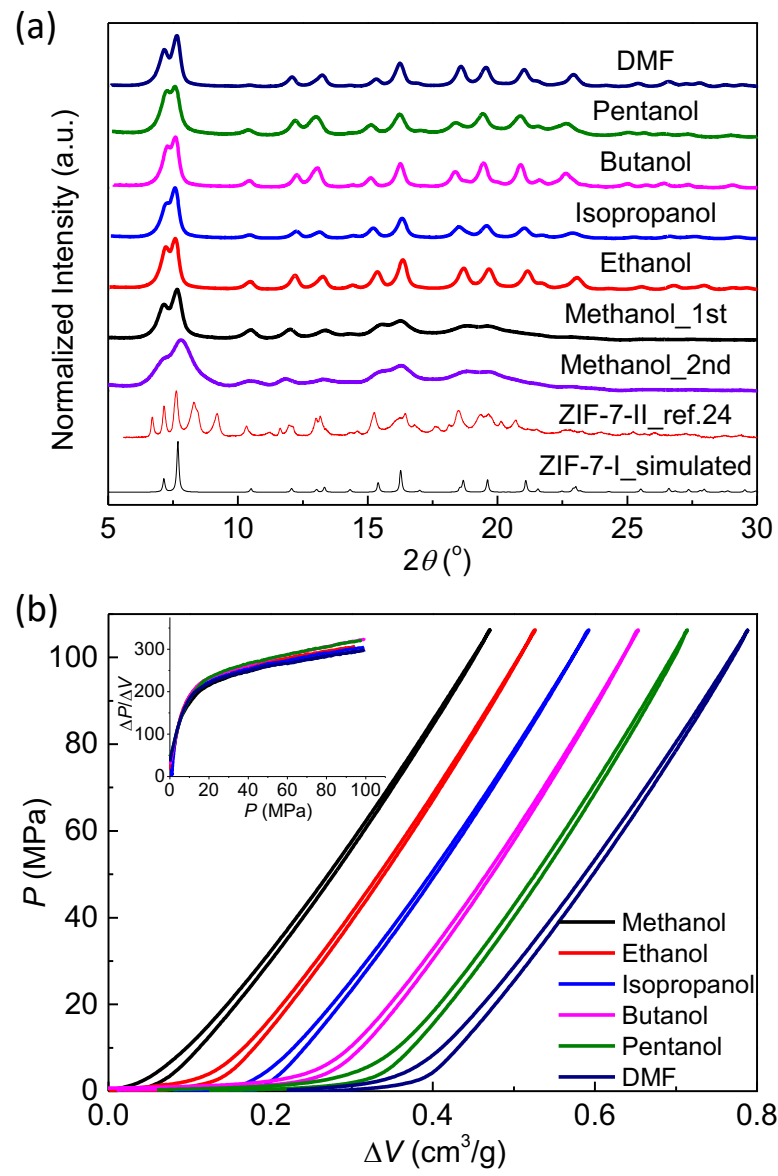

Figure 2. (a) XRD results of ZIF-7-I nanocrystals incorporating various solvent guest molecules. Two consecutive XRD scan results are presented to show the phase reversing back to ZIF-7-II (in air) due to the evaporation of methanol. (b) $P-\Delta V$ curves of water intrusion into ZIF-7-I nanocrystals incorporating various solvents. No intrusion plateau can be detected (inset), this means that the cavities of ZIF-7-I remain occupied by the solvent guests and cannot be intruded by water molecules even under mechanical pressure.

All of the above results concern the ZIF-7 nanocrystals. We anticipate that the crystal size of ZIF-7 may affect its liquid intrusion performance, in view of some previous studies on other nanoporous materials where the liquid intrusion pressure varies for different crystal sizes. ${ }^{40-41}$ To examine the crystal size effect, micron-sized ZIF-7 crystals were synthesized and their liquid intrusion tests were carried out, whose results are presented in Figure S4. However, none of their three phases can be intruded by water, including the guest-free ZIF-7-II. That is to say, water can only intrude the nanocrystals of ZIF-7-II. Given the ultrasmall window opening of ZIF-7 (that is smaller than the size of water molecules, see Figure S14), the inaccessibility of the bigger crystals may be linked to the lack of structural flexibility compared with the nanocrystals. Our results indicate that the crystal size effect is important, whereby the material near and on the outer surface of crystals is more flexible than the core ZIF-7 structure, and thus easier to undergo gate-opening which is a prerequisite for water intrusion to occur. Nanocrystals are therefore more preferable for water intrusion than its micronsized counterpart, having a larger area of crystal surface which interacts with the liquid molecules and favoring their intrusion. We confirmed that the ZIF-7-II nanocrystals have a significantly higher surface area through a Brunauer-Emmett-Teller (BET) test. Figure $\mathrm{S} 19$ shows the $\mathrm{N}_{2}$ physisorption results, and the corresponding external surface areas determined are: 18 $\mathrm{m}^{2} / \mathrm{g}$ for the micron-sized crystals and $70 \mathrm{~m}^{2} / \mathrm{g}$ for the nanosized crystals. This indicates that $\mathrm{N}_{2}$ is mainly physisorbed on the external surface of ZIF-7 crystals so that it's dependent on particle sizes, which is in agreement with a previous study. ${ }^{21}$ It is worth highlighting that major discrepancy has also been reported on the $\mathrm{CO}_{2}$ adsorption behavior of ZIF-7 with different crystal sizes, which has been ascribed to their different degrees of structural flexibility. ${ }^{21}$

\section{TUNING THE INTRUSION RESPONSE OF ZIF-7}

Based on the water intrusion behavior into the nanocrystals of ZIF-7-II, we anticipate that versatile systems can be developed with tunable intrusion pressure, energy dissipation and recoverability. As shown in Figure 3, intrusion pressure can be either decreased or increased by modifying the liquid properties. Here we demonstrate that alcohols (e.g., ethanol) may promote water intrusion while salts (e.g., lithium chloride, $\mathrm{LiCl}$ ) can inhibit water intrusion. Ethanol has a relatively small molecule size that can pass through ZIF-7's window apertures and its stronger affinity to ZIF-7 can lower the energy barrier of liquid intrusion. With the addition of ethanol, the decline of intrusion pressure is so significant that a completely spontaneous liquid inflow (i.e., $P_{\text {in }}=0 \mathrm{MPa}$ ) can be achieved at a concentration of around $10 \mathrm{wt} . \%$. On the contrary, $\mathrm{LiCl}$ serves as intrusion inhibitor which increases the intrusion pressure. It is striking to see that with $10 \mathrm{M}\left(\mathrm{mol} / \mathrm{kg}_{\mathrm{H}_{2} \mathrm{O}}\right) \mathrm{LiCl}$, the intrusion pressure soars up to $168 \mathrm{MPa}$, and the corresponding energy dissipation density has increased by more than a factor of three to yield 6.7 $\mathrm{J} / \mathrm{g}$ against the value of pure water intrusion $(2.1 \mathrm{~J} / \mathrm{g})$.

Furthermore, we discover that modification of the liquid phase can affect the extrusion performance as well. Intri- 
guingly, $\mathrm{LiCl}$ solution proves to function not only as an intrusion inhibitor, but also as an extrusion promoter. As shown in Figure $3 \mathrm{~b}$, by using $10 \mathrm{M} \mathrm{LiCl}$ solution, the intruded liquid will flow out of the framework upon unloading, in contrast to the non-outflow performance of pure water system. Subsequent loading cycles therefore still exhibit intrusion plateaus due to the recovery of ZIF-7 porosity after the preceding liquid extrusion cycle. On this basis, the employment of $\mathrm{LiCl}$ not only enhances the energy dissipation capacity, but also contributes to a new feature of the ZIF-7 intrusion system, namely, recoverability to enable sample reusability. Note that this finding validates the stability of ZIF-7 inside LiCl solution; it is important to note that some ZIF structures will collapse in electrolyte solutions. ${ }^{42}$

The effect of electrolytes on the water intrusion pressure has been demonstrated on some other nanoporous materials including zeolites, ${ }^{35,43-45}$ silica, ${ }^{46}$ as well as some MOFs ${ }^{42,47}$ However, the mechanism of how ions increase the water intrusion pressure is still not well understood. Some theories have been proposed, for example, the osmotic effect as a result of the ion exclusion during water intrusion (assuming that only water molecules penetrate the nanopores) ${ }^{47-48}$ the unique nano-crystallinelike structure of ion-couple inside the nanopores, ${ }^{49}$ and increased solid-liquid interfacial tension due to the inclusion of electrolytes. $^{35,46}$ Considering the narrow aperture of ZIF-7 framework, we propose that the additional osmotic pressure might be a primary cause for the intrusion inhibiting effect of $\mathrm{LiCl}$ in our experiments.

(a)
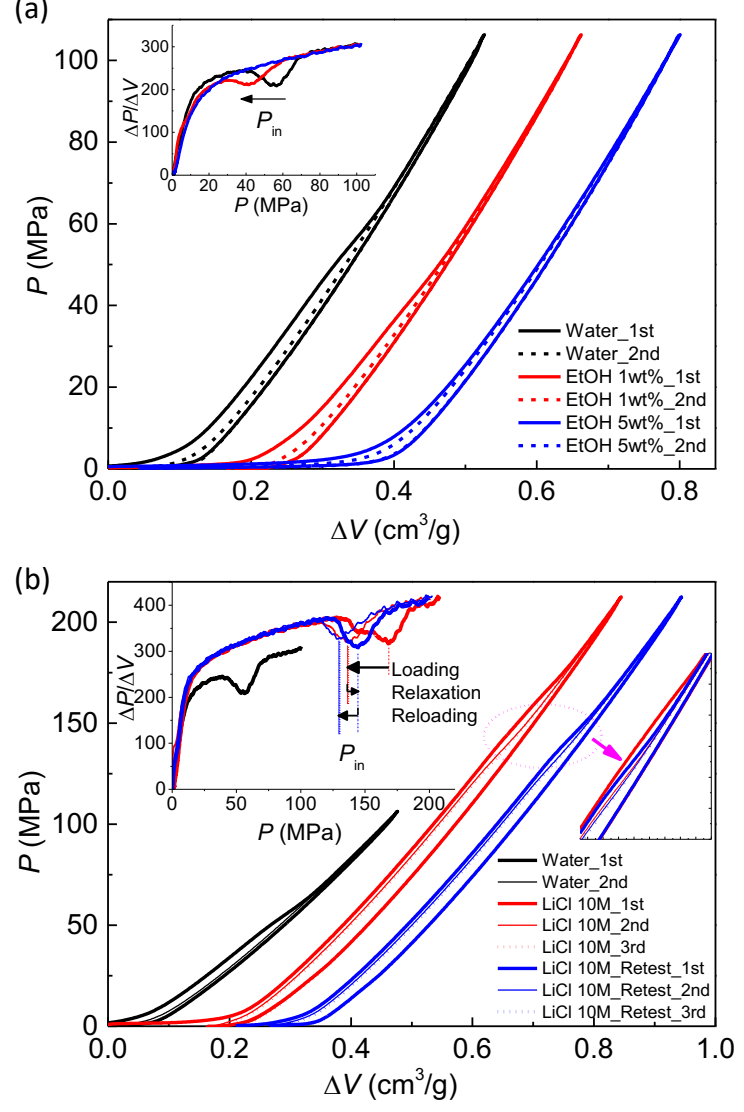

Figure 3. $P-\Delta V$ curves of liquid intrusion into ZIF-7-II nanocrystals with various liquid compositions. (a) Intrusion of ethanol $(\mathrm{EtOH})$ solutions at varying concentrations. (b) Intrusion of $\mathrm{LiCl}$ solutions at $10 \mathrm{M}\left(\mathrm{mol} / \mathrm{kg}_{\mathrm{H} 2 \mathrm{O}}\right)$. Two sets of 3-cycle measurements were performed with a relaxation time of $24 \mathrm{~h}$ apart. In the inset on the right, all the $P-\Delta V$ curves for $\mathrm{LiCl}(10 \mathrm{M})$ are stacked together to show the change of $P_{\text {in }}$ with multiple loading cycles and relaxation, which is further supported by the top-left inset showing the gradient $\Delta P / \Delta V$ of the first two cycles.

The cyclic intrusion of $\mathrm{LiCl}$ solution reveals the important viscoelastic structural deformation of ZIF-7-II nanocrystals upon the penetration of water molecules. As the top-left inset of Figure $3 \mathrm{~b}$ shows, the intrusion pressure in the second cycle (thin red line) drops from the initial intrusion pressure (thick red line), which can be explained by the expansion of the narrow channels interconnecting the ZIF-7 pores due to the preceding liquid intrusion. By virtue of the gate-opening mechanism upon mechanical pressure, larger windows become accessible in the subsequent loading cycle and thus a lower intrusion pressure was observed. Intriguingly, this residual deformation can be recovered through structural relaxation. When the $10 \mathrm{M} \mathrm{LiCl} \mathrm{so-}$ lution penetrates the same ZIF-7-II specimen again after an interval of $24 \mathrm{~h}$, the intrusion pressure can be partially recovered (thick blue line), indicating that the ZIF-7-II structure may recover to its initial state during relaxation with the 'gates closing'. Similarly, the reloading will open the gates again and reduce the intrusion pressure (thin blue line). From the above observation, we established that the mechanical property of ZIF-7 is viscoelastic, meaning that the gate-opening mechanism is indeed time-dependent and recoverable via structural relaxation.

\section{CONCLUSIONS}

In summary, we present the first successful study of liquid intrusion into ZIF-7 facilitated by gate-opening phenomenon. Between the three distinct phases and two crystal sizes, water intrusion is observed only on the guest-free ZIF-7-II nanocrystals. Though the cavity in ZIF-7-II is smaller than that in ZIF-7-I and has no apparent solvent accessibility, ${ }^{24}$ by using liquid intrusion technique, we demonstrate that ZIF-7-II becomes accessible to water molecules, and the water incorporation does not induce phase transition as other solvents do. Additionally, the gate-opening/-closing mechanism upon the penetration of water molecules reveals the viscoelastic behavior of the ZIF-7 framework, which is time-dependent and recoverable by structural relaxation. This study has advanced our understanding of the mechanical properties of ZIF-7, which is key to the design of practical applications.

This work proves that ZIF materials can be intruded by water even if their pore aperture size is smaller than water molecules, in contrast to the previous studies where larger apertures are provided such that water intrusion is possible even without any gate-opening. This unique gate-opening facilitated water intrusion phenomenon not only demonstrates the flexibility of ZIF structures, but also contributes to an exceptional non-outflow performance upon unloading, which is attractive for impact mitigation applications. We show that it is possible to harness this 'plastic' compressive deformation behavior, combined with its high intrusion pressure, to accomplish a substantial energy dissipation density despite its limited pore volume.

Moreover, we demonstrate several straightforward methods for tuning the intrusion pressure, energy dissipation and recoverability by manipulating the liquid properties, paving the way for innovative practical implementations. For example, the addition of ethanol promotes water intrusion, while $\mathrm{LiCl}$ can act 
as both intrusion inhibitors and extrusion promoters thereby enabling the ZIF-7 intrusion system to be reusable in multi-cyclic applications. This part of work can be extended to many other different kinds of liquids, and a systematic investigation on the influence of liquid properties on the gate-opening and intrusion behavior of ZIF-7 can be conducted in the future.

We found that the ZIF-7 materials are adequately stable against water intrusion in this work. However, considering the fact that ZIF-7-II will transform into the nonporous ZIF-7-III upon prolonged immersion in water, the long-term durability of ZIF-7+water systems should be systematically interrogated in future studies to enable real-world applications. New research opportunities and future engineering solutions may include the adoption of other combinations of intruding liquids or additives, crystal surface functionalization to enhance hydrophobicity, and exploitation of the crystal size effect.

\section{ASSOCIATED CONTENT}

Supporting Information. Materials synthesis and characterization techniques, supporting figures and additional electron microscopy images. This material is available free of charge via the Internet at http://pubs.acs.org.

\section{AUTHOR INFORMATION}

\section{Corresponding Author}

*J.C. Tan. E-mail: jin-chong.tan@eng.ox.ac.uk

\section{Author Contributions}

Y.S. and J.C.T. conceived the project. Y.S. designed and performed the experiments. Y.S. analyzed the data with input from J.C.T. Y.S. and J.C.T. prepared the manuscript. All authors contributed to the discussions of the results and have given approval to the final version of the manuscript.

\section{Funding Sources}

This work was funded by the K.C. Wong Fellowship.

\section{Notes}

The authors declare no competing financial interest.

\section{ACKNOWLEDGMENT}

Y. Sun would like to thank the K.C. Wong Fellowship for funding the research. We thank Prof. Clive Siviour at Oxford University for helpful discussions. We thank $\mathrm{Dr} \mathrm{Pu}$ Zhao from ref.24, for supplying the published XRD data of the micronsized crystals of ZIF-7-II. We are grateful to the ISIS Rutherford Appleton Laboratory, especially Dr. James Taylor at the R79 Hydrogen and Catalysis Laboratory for the BET measurement, and Dr. Gavin Stenning and Dr Marek Jura at the R53 Materials Characterization Laboratory for access to the XRD facilities. We thank the Research Complex at Harwell ( $\mathrm{RCaH})$ for providing access to the FTIR and Raman spectrometers. We thank Kirill Titov at Oxford University for the FEGSEM imaging.

\section{REFERENCES}

(1) Srivastava, A.; Srivastava, O.; Talapatra, S.; Vajtai, R.; Ajayan, P. Carbon Nanotube Filters. Nat. Mater. 2004, 3, 610-614.
(2) Martin, C. R.; Kohli, P. The Emerging Field of Nanotube Biotechnology. Nat. Rev. Drug Discov. 2003, 2, 29-37.

(3) Eroshenko, V.; Regis, R.-C.; Soulard, M.; Patarin, J. Energetics: A New Field of Applications for Hydrophobic Zeolites. J. Am. Chem. Soc. 2001, 123, 8129-8130.

(4) Desbiens, N.; Demachy, I.; Fuchs, A. H.; Kirsch - Rodeschini, H.; Soulard, M.; Patarin, J. Water Condensation in Hydrophobic Nanopores. Angew. Chem. Int. Ed. 2005, 44, 5310-5313.

(5) Chen, X.; Xu, B.; Liu, L. Nanoscale Fluid Mechanics and Energy Conversion. Appl. Mech. Rev. 2014, 66, 050803.

(6) Sun, Y.; Guo, Z.; Xu, J.; Xu, X.; Liu, C.; Li, Y. A Candidate of Mechanical Energy Mitigation System: Dynamic and Quasi-Static Behaviors and Mechanisms of Zeolite B/Water System. Mater. Des. 2015, $66,545-551$

(7) Fraux, G.; Coudert, F.-X.; Boutin, A.; Fuchs, A. H. Forced Intrusion of Water and Aqueous Solutions in Microporous Materials: From Fundamental Thermodynamics to Energy Storage Devices. Chem. Soc. Rev. 2017, 46, 7421-7437.

(8) Yot, P. G.; Vanduyfhuys, L.; Alvarez, E.; Rodriguez, J.; Itié, J.-P.; Fabry, P.; Guillou, N.; Devic, T.; Beurroies, I.; Llewellyn, P. L. Mechanical Energy Storage Performance of an Aluminum Fumarate Metal-Organic Framework. Chem. Sci. 2016, 7, 446-450.

(9) Grosu, Y.; Mierzwa, M.; Eroshenko, V. A.; Pawlus, S.; Chorażewski, M.; Nedelec, J.-M.; Grolier, J.-P. E. Mechanical, Thermal, and Electrical Energy Storage in a Single Working Body: Electrification and Thermal Effects Upon Pressure-Induced Water Intrusion-Extrusion in Nanoporous Solids. ACS Appl. Mater. Interfaces 2017, 9, 7044-7049.

(10) Tzanis, L.; Trzpit, M. 1.; Soulard, M.; Patarin, J. 1. Energetic Performances of Channel and Cage-Type Zeosils. J. Phys. Chem. C 2012, 116, 20389-20395.

(11) Qiao, Y.; Liu, L.; Chen, X. Pressurized Liquid in Nanopores: A Modified Laplace-Young Equation. Nano Lett. 2009, 9, 984-988.

(12) Mortada, B.; Chaplais, G.; Veremeienko, V.; Nouali, H.; Marichal, C.; Patarin, J. Energetic Performances of ZIF-8 Derivatives: Impact of the Substitution (Me, Cl, or Br) on Imidazolate Linker. J. Phys. Chem. C 2018, 122, 3846-3855.

(13) Majumder, M.; Chopra, N.; Andrews, R.; Hinds, B. J. Nanoscale Hydrodynamics: Enhanced Flow in Carbon Nanotubes. Nature 2005, 438, 44-44

(14) Holt, J. K.; Park, H. G.; Wang, Y .; Stadermann, M.; Artyukhin, A. B.; Grigoropoulos, C. P.; Noy, A.; Bakajin, O. Fast Mass Transport through Sub-2-Nanometer Carbon Nanotubes. Science 2006, 312, 1034 1037.

(15) Tinti, A.; Giacomello, A.; Grosu, Y.; Casciola, C. M. Intrusion and Extrusion of Water in Hydrophobic Nanopores. Proc. Natl. Acad. Sci. U. S. A. 2017, 201714796.

(16) Xu, B.; Li, Y.; Park, T.; Chen, X. Effect of Wall Roughness on Fluid Transport Resistance in Nanopores. J. Chem. Phys. 2011, 135, 144703.

(17) Tan, J. C.; Bennett, T. D.; Cheetham, A. K. Chemical Structure, Network Topology, and Porosity Effects on the Mechanical Properties of Zeolitic Imidazolate Frameworks. Proc. Natl. Acad. Sci. U. S. A. 2010, 107, 9938-9943.

(18) Tan, J. C.; Cheetham, A. K. Mechanical Properties of Hybrid Inorganic-Organic Framework Materials: Establishing Fundamental Structure-Property Relationships. Chem. Soc. Rev. 2011, 40, 1059-1080. (19) Phan, A.; Doonan, C. J.; Uribe-Romo, F. J.; Knobler, C. B.; O’keeffe, M.; Yaghi, O. M. Synthesis, Structure, and Carbon Dioxide Capture Properties of Zeolitic Imidazolate Frameworks. Acc. Chem. Res. 2010, 43, 58-67.

(20) Borjigin, T.; Sun, F.; Zhang, J.; Cai, K.; Ren, H.; Zhu, G. A Microporous Metal-Organic Framework with High Stability for Gc Separation of Alcohols from Water. Chem. Commun. 2012, 48, 76137615 .

(21) Cai, W.; Lee, T.; Lee, M.; Cho, W.; Han, D.-Y.; Choi, N.; Yip, A. C.; Choi, J. Thermal Structural Transitions and Carbon Dioxide Adsorption Properties of Zeolitic Imidazolate Framework-7 (ZIF-7). J. Am. Chem. Soc. 2014, 136, 7961-7971.

(22) Gücüyener, C.; van den Bergh, J.; Gascon, J.; Kapteijn, F. Ethane/Ethene Separation Turned on Its Head: Selective Ethane Adsorption on the Metal- Organic Framework ZIF-7 through a GateOpening Mechanism. J. Am. Chem. Soc. 2010, 132, 17704-17706.

(23) Khay, I.; Chaplais, G.; Nouali, H.; Ortiz, G.; Marichal, C.; Patarin, J. Assessment of the Energetic Performances of Various ZIFs with SO or 
RHO Topology Using High Pressure Water Intrusion-Extrusion Experiments. Dalton Trans. 2016, 45, 4392-4400.

(24) Zhao, P.; Lampronti, G. I.; Lloyd, G. O.; Wharmby, M. T.; Facq, S. b.; Cheetham, A. K.; Redfern, S. A. Phase Transitions in Zeolitic Imidazolate Framework 7: The Importance of Framework Flexibility and Guest-Induced Instability. Chem. Mater. 2014, 26, 1767-1769.

(25) Du, Y.; Wooler, B.; Nines, M.; Kortunov, P.; Paur, C. S.; Zengel, J.; Weston, S. C.; Ravikovitch, P. I. New High-and Low-Temperature Phase Changes of ZIF-7: Elucidation and Prediction of the Thermodynamics of Transitions. J. Am. Chem. Soc. 2015, 137, 13603-13611.

(26) Yang, Q.-F.; Cui, X.-B.; Yu, J.-H.; Lu, J.; Yu, X.-Y.; Zhang, X.; Xu, J.-Q.; Hou, Q.; Wang, T.-G. A Series of Metal-Organic Complexes Constructed from in Situ Generated Organic Amines. CrystEngComm 2008, 10, 1534-1541.

(27) Li, Y. S.; Liang, F. Y.; Bux, H.; Feldhoff, A.; Yang, W. S.; Caro, J. Molecular Sieve Membrane: Supported Metal-Organic Framework with High Hydrogen Selectivity. Angew. Chem. 2010, 122, 558-561.

(28) Tu, M.; Wiktor, C.; Rösler, C.; Fischer, R. A. Rapid Room Temperature Syntheses of Zeolitic-Imidazolate Framework (ZIF) Nanocrystals. Chem. Commun. 2014, 50, 13258-13260.

(29) Kang, C.-H.; Lin, Y.-F.; Huang, Y.-S.; Tung, K.-L.; Chang, K.-S.; Chen, J.-T.; Hung, W.-S.; Lee, K.-R.; Lai, J.-Y. Synthesis of ZIF7/Chitosan Mixed-Matrix Membranes with Improved Separation Performance of Water/Ethanol Mixtures. J. Membr. Sci. 2013, 438, 105111.

(30) Aguado, S.; Bergeret, G.; Titus, M. P.; Moizan, V.; Nieto-Draghi, C.; Bats, N.; Farrusseng, D. Guest-Induced Gate-Opening of a Zeolite Imidazolate Framework. New J. Chem. 2011, 35, 546-550.

(31) Park, K. S.; Ni, Z.; Côté, A. P.; Choi, J. Y.; Huang, R.; Uribe-Romo, F. J.; Chae, H. K.; O'Keeffe, M.; Yaghi, O. M. Exceptional Chemical and Thermal Stability of Zeolitic Imidazolate Frameworks. Proc. Natl. Acad. Sci. U.S. A. 2006, 103, 10186-10191.

(32) Rodriguez, J.; Beurroies, I.; Loiseau, T.; Denoyel, R.; Llewellyn, P. L. The Direct Heat Measurement of Mechanical Energy Storage MetalOrganic Frameworks. Angew. Chem. Int. Ed. 2015, 54, 4626-4630.

(33) Grosu, Y.; Gomes, S.; Renaudin, G.; Grolier, J.-P. E.; Eroshenko, V.; Nedelec, J.-M. Stability of Zeolitic Imidazolate Frameworks: Effect of Forced Water Intrusion and Framework Flexibility Dynamics. RSC Adv. 2015, 5, 89498-89502.

(34) Sun, Y.; Li, Y.; Tan, J.-C. Framework Flexibility of ZIF-8 under Liquid Intrusion: Discovering Time-Dependent Mechanical Response and Structural Relaxation. Phys. Chem. Chem. Phys. 2018, 20, 10108-10113. (35) Sun, Y.; Lu, W.; Li, Y. A Defiltration Control Method of Pressurized Liquid in Zeolite ZSM-5 by Silanol Introduction. Appl. Phys. Lett. 2014, $105,121609$.

(36) Sun, Y.; Li, P.; Qiao, Y.; Li, Y. Time-Dependent Gas-Liquid Interaction in Molecular-Sized Nanopores. Sci.Rep. 2014, 4, 6547.
(37) Ryder, M. R.; Civalleri, B.; Bennett, T. D.; Henke, S.; Rudić, S Cinque, G.; Fernandez-Alonso, F.; Tan, J.-C. Identifying the Role of Terahertz Vibrations in Metal-Organic Frameworks: From Gate-Opening Phenomenon to Shear-Driven Structural Destabilization. Phys. Rev. Lett. 2014, 113,215502 .

(38) Johan, E.; Abadal, C. R.; Sekulić, J.; Chowdhury, S. R.; Blank, D. H. Transport Mechanisms of Water and Organic Solvents through Microporous Silica in the Pervaporation of Binary Liquids. Microporous Mesoporous Mater. 2003, 65, 197-208.

(39) Bux, H.; Liang, F.; Li, Y.; Cravillon, J.; Wiebcke, M.; Caro, J. r. Zeolitic Imidazolate Framework Membrane with Molecular Sieving Properties by Microwave-Assisted Solvothermal Synthesis. J. Am. Chem. Soc. 2009, 131, 16000-16001.

(40) Khay, I.; Chaplais, G.; Nouali, H.; Marichal, C.; Patarin, J. Water Intrusion-Extrusion Experiments in ZIF-8: Impacts of the Shape and Particle Size on the Energetic Performances. RSC Adv. 2015, 5, 31514 31518.

(41) Sun, Y.; Xu, J.; Li, Y. B.; Liu, B.; Wang, Y.; Liu, C.; Chen, X. Experimental Study on Energy Dissipation Characteristics of ZSM - 5 Zeolite/Water System. Adv. Eng. Mater. 2013, 15, 740-746.

(42) Ortiz, G.; Nouali, H.; Marichal, C.; Chaplais, G. r.; Patarin, J. 1 Energetic Performances of "ZIF-71-Aqueous Solution" Systems: A Perfect Shock-Absorber with Water. J. Phys. Chem. C 2014, 118, 2131621322 .

(43) Ronchi, L.; Ryzhikov, A.; Nouali, H.; Daou, T. J.; Patarin, J Energetic Performances of Pure-Silica DDR Zeolite by High Pressure Intrusion-Extrusion of Electrolyte Aqueous Solutions: A Shock-Absorber with Huge Absorbed Energy. J. Phys. Chem. C 2018, 122, 2726-2733. (44) Sun, Y.; Xu, J.; Li, Y.; Xu, X.; Liu, C.; Chen, X. Mechanism of Water Infiltration and Defiltration through ZSM-5 Zeolite: Heating and Sodium Chloride Concentration Effect. J. Nanomater. 2013, 2013, 120. (45) Ryzhikov, A.; Nouali, H.; Daou, T.; Patarin, J. A Drastic Influence of the Anion Nature and Concentration on High Pressure Intrusion-Extrusion of Electrolyte Solutions in Silicalite-1. Phys. Chem. Chem. Phys. 2018, 20, 6462-6468.

(46) Surani, F. B.; Qiao, Y. Infiltration and Defiltration of an Electrolyte Solution in Nanopores. J. Appl. Phys. 2006, 100, 034311.

(47) Michelin-Jamois, M.; Picard, C.; Vigier, G.; Charlaix, E. Giant Osmotic Pressure in the Forced Wetting of Hydrophobic Nanopores. Phys. Rev. Lett. 2015, 115, 036101.

(48) Gupta, K. M.; Qiao, Z.; Zhang, K.; Jiang, J. Seawater Pervaporation through Zeolitic Imidazolate Framework Membranes: Atomistic Simulation Study. ACS Appl. Mater. Interfaces 2016, 8, 13392-13399. (49) Liu, L.; Chen, X.; Lu, W.; Han, A.; Qiao, Y. Infiltration of Electrolytes in Molecular-Sized Nanopores. Phys. Rev. Lett. 2009, 102, 184501 . 
Table of Contents

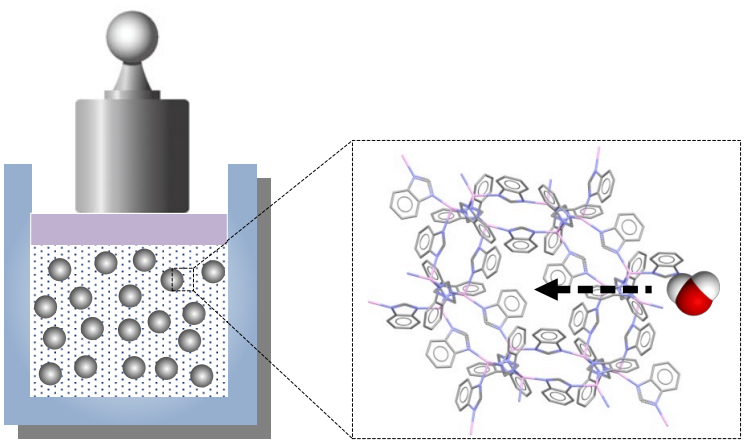

